\title{
Minimum Latency Data Aggregation in Wireless Sensor Network
}

\author{
Gagandeep Kaur \\ Student \\ Yadavindra College of \\ Engineering, Punjabi \\ University, Talwandi Sabo, \\ Punjab (India)
}

\author{
Sukhwinder Singh Sran \\ Assistant Professor \\ Yadavindra College of \\ Engineering, Punjabi \\ University, Talwandi Sabo, \\ Punjab (India)
}

\author{
Navjot Kaur \\ Assistant Professor \\ College of Engineering and \\ Management, Neighbourhood \\ Campus Punjabi University, \\ Rampura Phul, Punjab (India)
}

\begin{abstract}
The wireless sensor networks are highly constrained type of network having sensor nodes with more capabilities. The sensor networks are deployed in various regions to collect the data. The critical issue of wireless Sensor Networks (WSNs) are network life and latency incurred to report data, which is the main area of research nowadays. The proposed model is using an optimized localization technique for data aggregation and consists of various regional aggregators that aggregate data to reduce the energy consumption and helps to enlarge the lifespan of cluster head in our existing scheme. The proposed model has been designed using the regional aggregators which lower the routing overhead over the transit cluster heads cum routers in the path between the target cluster head and the sink node. Hence network lifetime of sensor nodes is increased. The proposed model has been proved to be efficient in case of performance parameters of transmission delay reduced by the factor of one-third. Similarly the proposed model shows better performance in terms of network load, throughput, packet delivery ratio etc. parameters. The experimental results have proved the efficiency of the proposed model in the real time applications.
\end{abstract}

\section{Keywords}

Data Aggregation, Wireless Sensor Networks, Latency, Clustering.

\section{INTRODUCTION}

Wireless Sensor Network as name suggests that it is a wireless system having small low power devices, called nodes. These nodes use different types of sensors for sensing their surrounding conditions and communicate throughout the network through a wireless medium. The number of sensor nodes may vary from hundreds to thousands. The features of these sensor nodes comprise low cost, small size, low power, multi-functionality (sensing, processing, routing etc.). The sensor nodes for such applications are usually designed to work in conditions where it may not be possible to recharge or replace the batteries of the nodes. This means that energy is a very precious resource for sensor nodes, and communication overhead is to be minimized.

Nowadays, WSN used in numerous applications like health, environment, space exploration, military surveillance and other commercial areas. The main characteristics of WSN include densely node deployment, random topology change, application specific etc. Sensor networks are event based systems. Sensor nodes will periodically sense their nearby environment and send the information to a sink which is not limited in energy. In any suggested geographical area, WSN nodes can be deployed and a base station is located inside or near the sensing region. The exchange of data between sensor nodes and base station can take place either in hierarchal or in flat manner. Hierarchical architecture also called clustering, where sensor nodes first grouped into a cluster and a node having high energy is selected from cluster as a cluster head. Sensor nodes transmit their data towards base station through different cluster heads. Every cluster head performs aggregation of data sent by neighbor nodes. Data aggregation is important in WSN because sensor nodes are densely deployed in wireless sensor network and large possibility that different nodes send same data to head. The aim of aggregation is to eliminate redundant data transmission and to enhance the lifespan of wireless sensor network.

Data aggregation is a habitual process of gathering or collecting the sensor data using aggregation approaches. In order to save resources and energy, data must be aggregated which also avoids traffic in the network. The main aim of data aggregation is to eliminate redundancy and enhances life time of network. Wireless sensor networks have limited computational power and limited memory and battery power. A data aggregation framework on wireless sensor networks is presented here.

The data is aggregated in three stages: Deployment, Data Delivery and Control Message Dissemination.

Clustering: Sensor nodes comprised of small powered battery, communication unit and transceiver. Nodes have limited energy and these cannot be recharged easily. So low power consumption is an important issue. Clustering is one of the important methods for prolonging the network lifetime in wireless sensor networks (WSNs). Clustering is grouping of sensor nodes(based on their transmission range) into clusters. In clustering, sensor nodes are grouped together to form cluster and a node having higher energy level is chosen as a cluster head(CH). Cluster head collects data from sensor nodes as well helps to control routs of data and schedule them. Clustering involves two phases.

Cluster Formation: During initial phase, whole nodes from network are arranged into groups and group of nodes is called cluster.

Cluster Head Selection: In this, based on criteria such that a node having higher energy is selected as a Cluster Head from each cluster.

There are different advantages of clustering:

- Cluster head perform aggregation and amount of redundant data can be mitigated. 
- Gives better throughput of network under high load also helps to minimize the collision of data.

\section{LITERATURE REVIEW}

A literature review goes beyond the search for information and includes the identification and articulation of relationships between the literature and our field of research. While the form of the literature review may vary with different types of studies, the basic purposes remain constant:

X. Chen et al. [1] Many applications of Collecting data from sensor nodes in wireless sensor networks over large geometrical domain imposed stringent delay needs. The Minimum Data Aggregation problem gives schedule which has shortest time for gathering data to sink node and route of data to be aggregated. An approximation algorithm was designed for MDAT problem. S.C.H.Huang et al. [4] In synchronous multi-hop wireless networks seeks a MLAS for data aggregation. Every node having a unit communication as well interference radius $\rho \geq 1$. Best aggregation latency with $\rho$ $=1$ is $23 \mathrm{R}+\Delta-18, \mathrm{R}$ is radius and $\Delta$ maximum degree of communication topology. With $\rho=1$, three aggregation schedules are constructed having latency $15 \mathrm{R}+\Delta-4,2 \mathrm{R}+$ $\mathrm{O}(\log \mathrm{R})+\Delta$ and $(1+\mathrm{O}(\log \mathrm{R} / 3 \sqrt{\mathrm{R}})) \mathrm{R}+\Delta$ respectively. X.Y.Li et al. [6] Reduce energy cost and minimize the time duration (Latency) both are studied for WSNs. Algorithms having theoretical performance are only for protocol interference model. This paper gives design of time efficient aggregation algorithm under physical interference model. Algorithm produces a collision-free aggregation schedule and data aggregation tree. Paper focuses on latency of aggregation schedule bounded by $\mathrm{O}(\mathrm{R}+\Delta)$ time slots. $\Delta$ is maximal node degree and $\mathrm{R}$ is network radius. Latency acquired by time efficient aggregation algorithm matches lower-bound for random wireless networks. B.Yu et al. [8] Paper gives minimum-time aggregation scheduling problem in multi-sink sensor networks (MS-MTAS problem). Work on MS-MTAS is NP-hard. After presenting Lower bound, Maximal independent sets based approximation algorithms for MSMTAS are designed. Both are implemented by simulation and verify effectiveness of algorithms. Upper bounds of aggregation time are $(\min \{26 \mathrm{k}-2,2 \Delta\})$ and $\left(23 \max ^{\mathrm{k}}\right.$ $i=1\{R\}+2 \Delta-23$, where $k$ is the number of sinks. M. Kyung $\boldsymbol{e t}$ al. [10] Main point here is How to build effective schedules of data aggregation without any interference. Paper gives study of MLAS problem in signal interference-noise-ratio(SINR) physical model. Two constant factor approximation algorithms having latency bound OðDpRP for double power model, d is max. Node degree of network. H. Li et al. [11] Protocol interference model fails of being an accurate abstraction of wireless interferences in real life. On contrary, physical interference model having potential to elevate network capacity. A distributed algorithm designed for wireless sensor networks of arbitrary topologies, aims to dwindle aggregation latency. $\mathrm{O}(\mathrm{k})$ slots are required for completion of aggregation task, $\mathrm{k}$ is logarithm of ratio between lengths of shortest and longest links in network. Partitioning of network into cells is done according to value of $\mathrm{k}$. A centralized algorithm proposed to construct aggregation tree following a nearest-neighbor criterion, with time slots $\mathrm{O}(\log \mathrm{n})$ and $\mathrm{O}\left(\log ^{3} \mathrm{n}\right)$ when coupled with link scheduling methods.

\section{METHODOLOGY}

The research methodology focus on cluster based networks. To deal with the problem of high transmission power and delay, a technique called optimized localization technique is proposed i.e. we will elect different regional leaders varying form cluster to cluster to mitigate higher energy consumption at node working as cluster head. These regional leaders will aggregate data from their neighboring nodes and transmit after aggregation towards cluster head. Cluster Head will drop the copies of data packets that are already received i.e. will help to maintain data integrity throughout the network. Then aggregated data can be forwarded towards base station. Data redundancy can be lessened by our proposed architecture and will help to enlarge lifespan of WSN cluster.

\section{Algorithm 1: Working of Regional Aggregator Model}

1. A geographic location is mapped and analyzed before deployment.

2. A sensor topological formation is selected for the region before deployment.

3. In case a Base station is located at top, the hierarchical topology formation is used to deploy sensor.

4. Power on the Wireless Sensor Network.

5. Form the cluster in the WSN topology.

6. Select the cluster head.

7. Non-dominating nodes within a cluster forward their data to the dominating node i.e. Cluster $\operatorname{Head}(\mathrm{CH})$.

8. Cluster head will generate an aggregation stream by collaborating the data from the non-dominating nodes in region.

9. Regional aggregator or Cluster Head will forward data toward the next level cluster Head in the WSN topology in experiment.

10. Next Level Cluster Head or Regional aggregator will aggregate the data from two streams:

Local Stream will be formed by data obtained from non-dominating nodes in the cluster region and Preaggregated Stream is the aggregated data coming from regional Cluster Head in lower hierarchy.

11. Ongoing regional aggregator will create a data aggregate from both of the streams (Local and Pre-aggregated).

\section{COMPARATIVE ANALYSIS}

Transmission Delay: Delay is the time required to transmit the packet from one node to another node. The delay is measured in seconds that how many seconds the source node is taking to send data to the sink node.

As Shown in figure, $\mathrm{X}$-axis shows time(seconds) and Y-Axis gives Delay in miliseconds. In Improved Minimum Latency Aggregation(IMLA), simulation delay has been computed for simulation period of 10 seconds. The recording interval is set at every 0.5 second. 


\section{Transmission Delay}

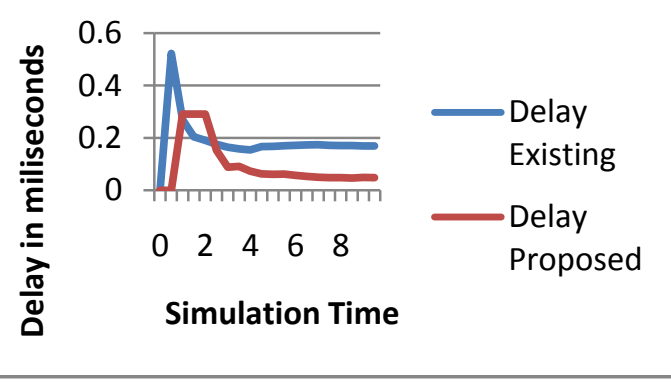

Figure 1 Transmission delay based comparison

The proposed and existing model results have been presented in the figure above for results of transmission delay. Performance parameter transmission delay indicates the time taken by a data packet travelling from source and destination nodes. The IMLA scheme offers the average latency of almost 0.03 seconds for the data aggregation under the simulation of 20 nodes. Thus graphical representation shows better performance than MLA(existing scheme).

Load: Refers to the amount of data being processed by the network. It is typically measured in Kbps. The routes are set up within the clusters which help to reduce the size of the routing table stored at the individual sensor nodes, i.e. traffic on different nodes.

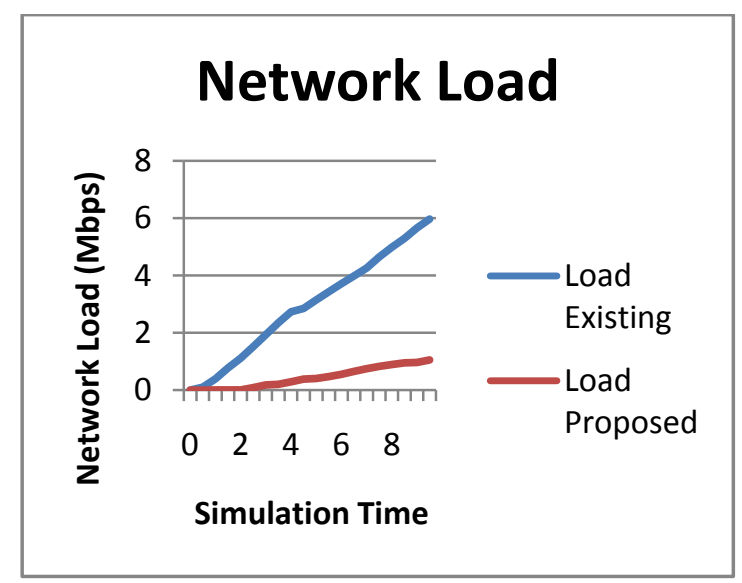

Figure 2 Network Load based comparison

As Shown in figure, simulation time is given along with $\mathrm{X}$ axis and Y-Axis gives Load in kbps. In the proposed model, simulation data load has been computed for simulation period of 10 seconds. After every 0.5 seconds, network load can be observed. In Improved MLA(Minimum Latency Aggregation) average network load is calculated 0.43 vis-à-vis 2.9 in MLA. In our simulated scheme, minimum and maximum network load recorded is $0.01 \mathrm{kbps}$ and $1.05 \mathrm{kbps}$ respectively in contrast to minimum( $0.09 \mathrm{kbps})$ and maximum(5.9kbps) values of network load in existing i.e. MLA technique. Hence IMLA gives better performance and helps to mitigate data or routing overhead.

Packet Loss: Packet loss is the breakdown of single or more delivered packets to reach their destination. This incident can cause visible effects in all digital infrastructure. The causes of packet loss comprise of insufficient signal power at the destination, usual or human made obstruction, extreme system sound, hardware crash, software sleaze or overwhelmed network nodes. Habitually more than one of these factors are concerned. In a case where the cause cannot be desired, packet loss hiding may be used to diminish the consequence of misplaced packets.

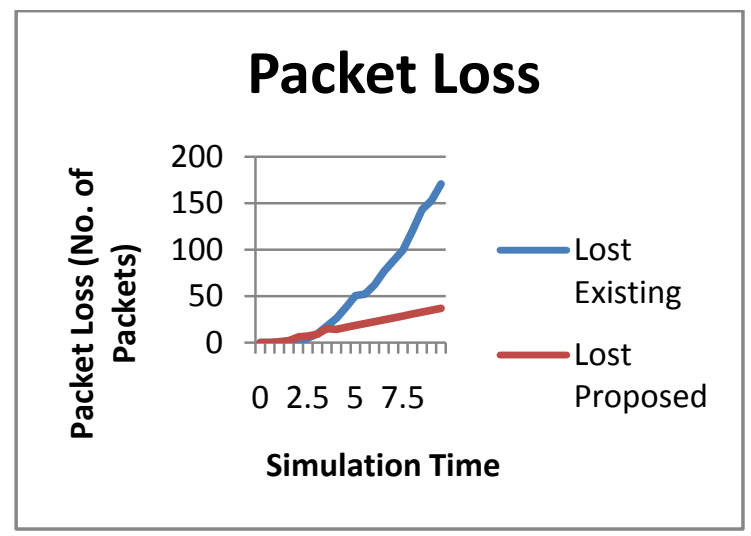

Figure 3 Packet loss existing v/s proposed

As Shown in figure, simulation time is given along with $\mathrm{X}$ axis and Y-Axis gives amount of lost data packets in kbps. In the proposed model, amount of lost data has been computed for simulation period of 10 seconds. Improved MLA gives minimum packet loss of zero packets. In new technique, average number of packets lost is approx. 17.3 which is reduced by factor of one-third vis-à-vis existing MLA scheme. So, objective of dissertation is fulflled.

Packet Delivery Ratio: Ratio of number of packets received by the node to the number of packets transmitted by the node. If PDR is greater in value it means protocol or network is good in performance. This parameter helps to measure the efficiency of our system's working. It is measured in number of packets sent.

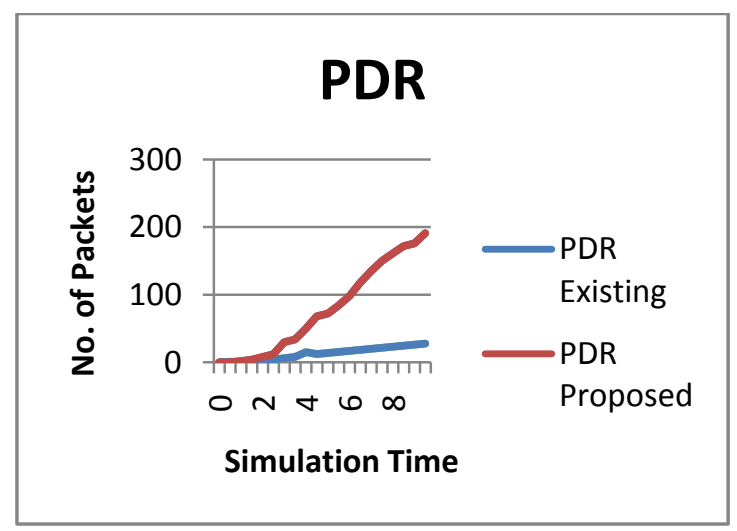

Figure 4 Packet Delivery Ratio based comparison

Figure presents that simulation time is given along with $\mathrm{X}$ axis and Y-Axis gives number of Packets. In proposed simulation model,we have considered simulation span of 10 seconds. After every 0.5 seconds, packet delivery ratio has been displayed. Throughout whole simulation period, minimum 2 packets delivered and 191 packets maximum. Average number of packets delivered are calculated from all the transactions is 78.2 . 
Table 1. Comparison Table

\begin{tabular}{|c|c|c|c|c|c|c|}
\hline \multirow[t]{2}{*}{$\begin{array}{c}\text { Parame } \\
\text { ters }\end{array}$} & \multicolumn{3}{|c|}{$\begin{array}{c}\text { Existing } \\
\text { Technique(Min. } \\
\text { Latency Aggregation) }\end{array}$} & \multicolumn{3}{|c|}{$\begin{array}{l}\text { Proposed Technique } \\
\text { (Improved Min. } \\
\text { Latency Aggregation ) }\end{array}$} \\
\hline & $\begin{array}{l}\text { Mini } \\
\text { mum }\end{array}$ & $\begin{array}{l}\text { Maxi } \\
\text { mum }\end{array}$ & $\begin{array}{l}\text { Aver } \\
\text { age }\end{array}$ & $\begin{array}{l}\text { Mini } \\
\text { mum }\end{array}$ & $\begin{array}{l}\text { Maxi } \\
\text { mum }\end{array}$ & $\begin{array}{c}\text { Aver } \\
\text { age }\end{array}$ \\
\hline $\begin{array}{c}\text { Transm } \\
\text { ission } \\
\text { Delay }\end{array}$ & 0.0 & 0.52 & 0.19 & 0.0 & 0.29 & 0.03 \\
\hline $\begin{array}{l}\text { Networ } \\
k \text { Load }\end{array}$ & 0.09 & 5.9 & 2.9 & 0.01 & 1.05 & 0.43 \\
\hline $\begin{array}{c}\text { Packet } \\
\text { Loss }\end{array}$ & 0 & 170.8 & 61.9 & 0 & 36.7 & 17.3 \\
\hline PDR & 1 & 27.6 & 13.1 & 2 & 191 & 78.2 \\
\hline $\begin{array}{c}\text { Throug } \\
\text { hput }\end{array}$ & 0.02 & 10.3 & 3.59 & 0.0 & 22.7 & 6.61 \\
\hline
\end{tabular}

Throughput: Throughput is the number of bits transfer per second. In wireless sensor networks throughput is the rate of production of something or the rate of processing The amount of data transferred from one place to another or processed in a specified amount of time. It is typically measured in Kbps, Mbps.

In Figure 5 throughput has been evaluated for simulation period of 10seconds is presented. After every 0.5 seconds, values can be observed. Throughout whole simulation process in existing(MLA) values computed for minimum and maximum throughput are $0.02 \mathrm{kbps}$ and $10.3 \mathrm{kbps}$ respectively. Average throuput calculated from all the transactions is $03.59 \mathrm{kbps}$. On the other hand, in improved technique(IMLA) the values calculated are 6.61 and 22.77 for average and maximum value respectively.objective is gained.

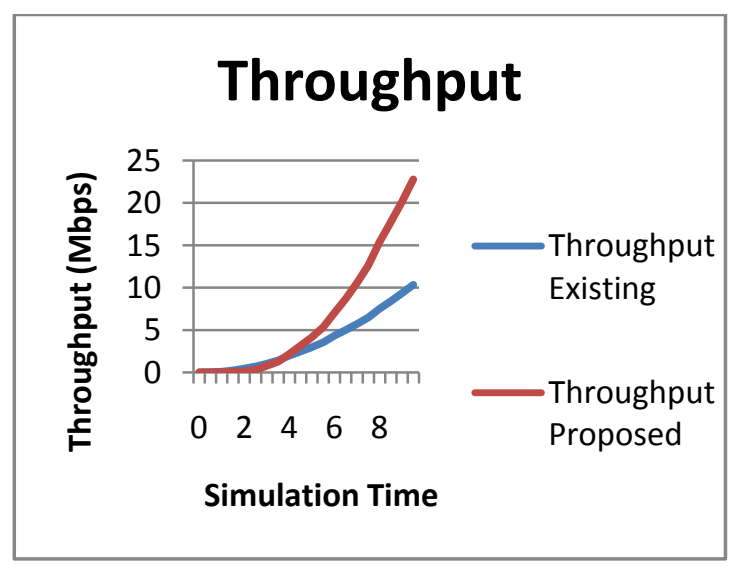

Figure 5 Throughput based comparison

\section{CONCLUSION}

The proposed model has been based upon the aggregation model for the sensor network. The proposed offers the sensor network connectivity in the multi-cluster formation in the hierarchical model. The newly designed aggregation model has been designed as the primary functional improvement in the existing systems of aggregations for the wireless sensor networks. The proposed model offer the use of regional aggregators to reduce the aggregation overhead from the main aggregator in order to make the sensor networks more efficient in terms of latency and sensor network lifetime. The proposed model has been tested over the sensor network simulated using the network simulator 2 (NS-2). The proposed model has been evaluated in the form of various performance parameters of network load, transmission delay, throughput, etc. The experimental results have shown the effectiveness of the proposed model for the sensor network lifetime elongation and routing overhead reduction in order to improve the latency in the packet delivery.

\section{FUTURE SCOPE}

In the future, the proposed model can be enhanced for the security using the multi-level authentication scheme or energy efficient tunneling mechanism for the wireless sensor network. The regional aggregators can be empowered to make the dynamic paths using the decentralized routing process. The proposed model can also be enhanced for the link level security between the regional aggregator and the primary aggregator in the given region of the sensor networks.

\section{REFERENCES}

[1] J. Zhu, X. Hu, X. Chen, "Minimum data aggregation time problem in wireless sensor networks", pp.133-142, IEEE, 2005.

[2] O.D. Incel, A. Ghosh, B. Krishnamachari, K. Chintalapudi, "Fast data collection in tree-based wireless sensor networks", theoretical aspects of distributed computing in sensor networks, pp.407-445, 2008.

[3] O. Goussevskaia, T. Moscibroda, R. Wattenhofer, "Local broadcasting in the physical interference model," in Proc. of dialm-pomc, 2008.

[4] P.J. Wan, S.C.H. Huang, L. Wang, Z. wan, X.Jia, "Minimum-latency aggregation scheduling in multihop wireless networks", in Proc. of MobiHoc, ACM, pp.185193, 2009.

[5] O. Goussevskaia, R. Wattenhofer, M.M. Halldorsson, E. Welzl, "Capacity of arbitrary wireless network," in Proc. of INFOCOM, IEEE, 2009.

[6] X.Y. Li, X. Xu, S. Wang, S. Tang, G. Dai, J. Zhao,Y. Qi, "Efficient data aggregation in multi-hop wireless sensor networks under physical interference model", pp. 353362, IEEE, 2009.

[7] Hongxing Li, Q.S. Hua, C. Wu, "Minimum-latency aggregation scheduling in wireless sensor networks under physical interference model", in Proc. of Modeling, Analysis and Simulation of Wireless and Mobile Systems(MSWiM), 2010.

[8] B. Yu and J.Li, "Minimum-time aggregation scheduling in multi-sink sensor networks", $8^{\text {th }}$ annual IEEE communications society conference on sensor, mesh and ad-hoc communications and networks, pp. 422-430, 2011. 
[9] L. Guo, Y. Li, S.K. Prasad, “An energy-efficient distributed algorithm for minimum-latency aggregation scheduling in wireless sensor networks", in Proc. of IEEE International Conference on Distributed Computing Systems, pp. 827-836, 2011.

[10] M. Kyung An, Nhat X. Lam, Dung T. Huynh, "Minimum latency data aggregation in the physical interference model", in Proc. of Ad Hoc Networks, pp. 2175-2186, 2012.

[11] H. Li, C. Wu, Q. S. Hua, F. C.M. Lau, "Latencyminimizing data aggregation in wireless sensor networks under physical interference model", in Proc. of Ad Hoc Networks, pp. 52-68, 2014.
[12] S. K. Gupta and P. Sinha, "Overview of Wireless Sensor Network: A Survey", in Proc. of International Journal of Advanced Research in Computer and Communication Engineering Vol. 3, 2014.

[13] M. Bhandari, S. Patil, T. Raju, "A Review on Efficient and Secure transmission of data for Cluster-Based Wireless Sensor Networks", in Proc. of International Journal of Innovative research in Science, Engineering and Technology, Vol. 3, pp. 9328-9332, 2014.

[14] F. Gielow , G. Jakllari , M. Nogueira , A. Santos, "Data Similarity aware dynamic node clustering in wireless sensor networks", in Proc. of Computer Networks, pp. 29-45, 2015. 